\title{
APLICAÇÃO DA CROMATOGRAFIA POR EXCLUSÃO E DA CROMATOGRAFIA GASOSA DE ALTA RESOLUÇÃO NA ANÁLISE DO CAFÉ
}

\author{
R. F. A. Moreira e L. C. Trugo* \\ Departamento de Bioquímica - Instituto de Química - Universidade Federal do Rio de Janeiro (UFRJ) \\ C. A. B. de Maria \\ Departamento de Ciências Fisiológicas - Instituto Biomédico - Universidade do Rio de Janeiro (UniRio)
}

\begin{abstract}
APPLICATION OF EXCLUSION CHROMATOGRAPHY AND HIGH RESOLUTION GAS CHROMATOGRAPHY TO COFFEE ANALYSIS. The combination of high performance exclusion chromatography (HPEC) and gas chromatography (GC) was applied to the analysis of six coffee samples that were previously characterized by sensory tests as of good or poor quality. The data obtained by the two techniques were statistically evaluated by "Principal Components Analysis" (PCA) using selected peak areas. The results showed the potential of the described techniques for coffee analysis. The HPEC technique monitored with the U.V. detector at $272 \mathrm{~nm}$ and followed by PCA may be correlated with sensorial data, particularly if a wider group of samples is used.
\end{abstract}

Keywords: coffee quality; high performance exclusion chromatography; principal components analysis.

\section{INTRODUÇÃO}

O café é reconhecido mundialmente pela grande receptividade que tem sua bebida, sendo considerado um dos produtos de maior comercialização no mercado internacional. Este grão possui particular importância para o Brasil, pois seu cultivo e industrialização geram um grande número de empregos e divisas para o país. Ainda hoje, o Brasil é reconhecido como um dos maiores produtores e exportadores mundiais de grãos de café e de café processado. Todos esses fatores citados anteriormente, deixam claro que estudos relacionados à composição do café, detecção de fraudes e avaliação de sua qualidade são indiscutivelmente importantes.

A qualidade do café está diretamente relacionada com suas características organolépticas. A baixa qualidade desse produto pode ser atribuída a espécies e variedades menos nobres, a técnicas inadequadas de colheita e/ou de processamento ou, ainda, a adição de substâncias que não fazem parte de sua composição natural. Atualmente, a avaliação da qualidade do café é realizada com indivíduos especialmente treinados, através da análise sensorial. A competitividade internacional tem exigido padrões de qualidade cada vez mais rigorosos. Dessa forma, torna-se crescente à busca de novas metodologias capazes de diferenciar, de forma prática e rápida, um café de boa qualidade de um de qualidade inferior. Dentre as novas metodologias que têm surgido, há uma tendência para a aplicação de técnicas de cromatografia gasosa de alta resolução (CGAR) e / ou da cromatografia líquida com fase reversa como métodos complementares à análise sensorial ${ }^{1}$.

A CGAR é muito empregada na análise dos componentes do aroma e, neste caso, os métodos de "headspace" são preferencialmente usados, por serem geralmente mais rápidos, simples e por fornecerem perfis de compostos voláteis mais genuínos do que as técnicas de destilação, já que a possibilidade de formação de artefatos é menor ${ }^{2,3}$. Dessa forma, as técnicas de "Headspace"-CGAR fornecem resultados representativos do

\footnotetext{
* Envio de correspondência: Departamento de Bioquímica, Instituto de Química, Universidade Federal do Rio de Janeiro, C. T. Bloco A, Cidade Universitária, 21949 - 900 Rio de Janeiro, Brasil. Fax: (021)290-4746
}

atributo aroma. Já as técnicas envolvendo a cromatografia líquida de alta eficiência fornecem resultados mais representativos do atributo sabor.

No presente trabalho, foi usada uma combinação de dados, obtidos pelo emprego da cromatografia por exclusão de alta eficiência (CEAE) e da técnica de "Headspace"-CGAR, para avaliar a qualidade de seis amostras de café após tratamento estatístico pelo método "Principal Components Analysis PCA". As características organolépticas destas amostras foram previamente determinadas por análise sensorial na própria indústria fornecedora das mesmas.

\section{PARTE EXPERIMENTAL}

\subsection{Amostras}

A pesquisa foi realizada com seis amostras de café verde, originárias de diferentes países, gentilmente cedidas pela empresa ILLYCAFFÈ (Itália). Estas amostras foram classificadas, através da análise sensorial, em A, B e C (amostras de boa qualidade) e D, E e F (amostras de qualidade inferior).

\subsection{CEAE}

Para as análises na CEAE, as amostras de café verde desengorduradas foram moídas em um moinho com malha de $0,75 \mathrm{~mm}$. Em seguida, realizou-se a extração de $0,5 \mathrm{~g}$ de cada amostra com água bidestilada a $80^{\circ} \mathrm{C}$ por 15 minutos em um banho com agitação. Na sequência, as amostras foram resfriadas à temperatura ambiente e, logo depois, filtradas com papel-de-filtro Whatman 42 para balões volumétricos de $50,0 \mathrm{ml}$. $\mathrm{O}$ volume destes balões foi ajustado com água bidestilada e, então, as soluções foram utilizadas para a cromatografia.

O trabalho foi realizado com dois sistemas isocráticos de CLAE. No primeiro, foi utilizado um cromatógrafo Shimadzu modelo LC-10AD (Japão) com bomba, detector por U.V. (0,16 AUFS e em $272 \mathrm{~nm}$ ) e válvula injetora Rheodyne (E.U.A.) com alça externa de $20 \mu \mathrm{l}$. A separação foi efetuada com uma coluna do tipo TSK G-3000 SW (300 mm de comprimento x 8 $\mathrm{mm}$ de d.i.) com sua respectiva pré-coluna (Supelco, E.U.A.) acoplada. A fase móvel foi composta de água bidestilada com $0,05 \%$ de azida de sódio e a vazão utilizada foi de $0,5 \mathrm{ml} / \mathrm{min}$.. 
No segundo sistema utilizou-se um detector por índice de refração diferencial (sensibilidade 64 e fator de escala 40) (Waters, E.U.A.). A coluna empregada neste estudo, bem como todos os demais equipamentos e condições cromatográficas foram idênticas as do outro sistema que utilizou o detector por U. V..

\section{3. "Headspace" - CGAR}

A análise de "Headspace" - CGAR foi efetuada de acordo com o método descrito por De Maria et $a l^{4}$. Neste método, 0,5 g de cada amostra moída de café verde desengordurado foi torrada a $220^{\circ} \mathrm{C}$ por 14 minutos em tubo de ensaio fechado, contendo septo $\mathrm{n}^{\mathrm{o}} 10043$ (Chrompack, Holanda). As amostras foram submetidas à vácuo, previamente à torrefação e, imediatamente após esse tratamento térmico, $4 \mathrm{ml}$ da fase gasosa produzida no interior do tubo foram coletados com uma seringa "gastight" (Hamilton, E. U. A.), através do septo e injetados no cromatógrafo.

A cromatografia gasosa foi realizada com um cromatógrafo Carlo Erba (Itália) modelo FTV série 4300. Utilizou-se uma coluna capilar de sílica com fase ligada Supelcowax 10, de 30 $\mathrm{m} \times 0,25 \mathrm{~mm}$ d.i. (Supelco, E.U.A.). As análises foram realizadas com taxa de divisão de 1:20, temperatura do detector por ionização em chama de $280^{\circ} \mathrm{C}$, temperatura do injetor de $260^{\circ} \mathrm{C}$ e uma velocidade linear de $40 \mathrm{~cm} / \mathrm{s}$ para o gás de arraste (hidrogênio). De acordo com a programação utilizada, o forno permaneceu por 10 minutos na temperatura inicial de $40^{\circ} \mathrm{C}$, em seguida, a temperatura foi acrescida de $3^{\circ} \mathrm{C}$ a cada minuto até que fosse atingida a temperatura final de $190^{\circ} \mathrm{C}$, onde permanecia por mais 15 minutos.

\subsection{Análise Estatística dos Resultados}

Para analisar os resultados obtidos através dos métodos cromatográficos empregados, utilizou-se um método estatístico conhecido como "Principal Components Analysis" (PCA). Este método permite que variáveis altamente correlacionadas sejam determinadas, de modo que a variabilidade de um sistema complexo possa ser expressa por estas variáveis, tão bem quanto seria se usássemos o conjunto das variáveis originais (Hewlett - Packard Corporation, 1986) ${ }^{5}$.

\section{RESULTADOS E DISCUSSÃO}

A figura 1(a) é representativa do perfil cromatográfico de um café verde de qualidade inferior, obtido por CEAE através de monitoração com detector por ultra-violeta em um comprimento de onda de $272 \mathrm{~nm}$. Como este cromatograma foi obtido pela técnica de exclusão, o fracionamento das substâncias ocorreu por diferença de massa molecular. As moléculas de maior massa molecular são eluídas mais rapidamente do que as moléculas que apresentam massas moleculares menores. A coluna TSK G3.000 SW, com limite de separação por massa molecular em torno de 1.000 a 300.000 daltons, foi padronizada, utilizando-se padrões de proteínas. Dessa forma, pode-se dividir este cromatograma em duas regiões distintas. A primeira região compreende os compostos de alta massa molecular. Esta região compreende compostos com massas moleculares superiores a 1.2 x 104 daltons (até 21 minutos). A resolução dessa região é menor do que a resolução da segunda região (21 até $40 \mathrm{~min}$.). A segunda região compreende as substâncias de baixa massa molecular. Podemos destacar, nesta segunda região, a presença de três importantes substâncias, presentes no café, que são o ácido clorogênico, a trigonelina e a cafeína, correspondendo respectivamente aos picos $\mathrm{A}$, $\mathrm{B}$ e $\mathrm{C}$, conforme descrito por De Maria et al e confirmado neste trabalho. Segundo esses autores, a separação entre estas três substâncias envolve, além do fenômeno convencional de exclusão, interações hidrofóbicas. Isto poderia explicar, inclusive, a melhor resolução da segunda região em relação a primeira no cromatograma. A figura 1(b) também corresponde a um cromatograma característico daquele mesmo café verde de qualidade inferior obtido por CEAE. Neste caso, porém, utilizou-se um detector de índice de refração diferencial. Este tipo de detector é muito utilizado para monitorar carboidratos em amostras de café. Este cromatograma apresenta um perfil bem distinto do anterior, no que diz respeito tanto a região de alta como de baixa massa molecular. A figura 2 apresenta um perfil cromatográfico característico de um café torrado de qualidade inferior obtido por "Headspace"-CGAR. Este cromatograma apresenta uma grande quantidade de picos, que embora não tenham sido identificados, mostraram claramente a complexidade da mistura. Os resultados obtidos através das três técnicas cromatográficas citadas ("Headspace"-CGAR e CEAE-UV e IR) foram analisados estatisticamente, baseando-se nas áreas dos respectivos picos. Antes, porém, decidiu-se
Figura 1. Perfis cromatográficos de um café verde de qualidade inferior (amostra D). (a) Obtido por CEAE-U.V. [(A) - ácido clorogênico (total), (B) - trigonelina, (C) - cafeína]; (b) Obtido por CEAE-I.R.. Coluna TSK G3.000 SW, fase móvel água bidestilada com $0,05 \%$ de azida de sódio e vazão de 0,5 ml/min. Os picos 1,2 e 3 sofreram tratamento estatístico através do "PCA". 


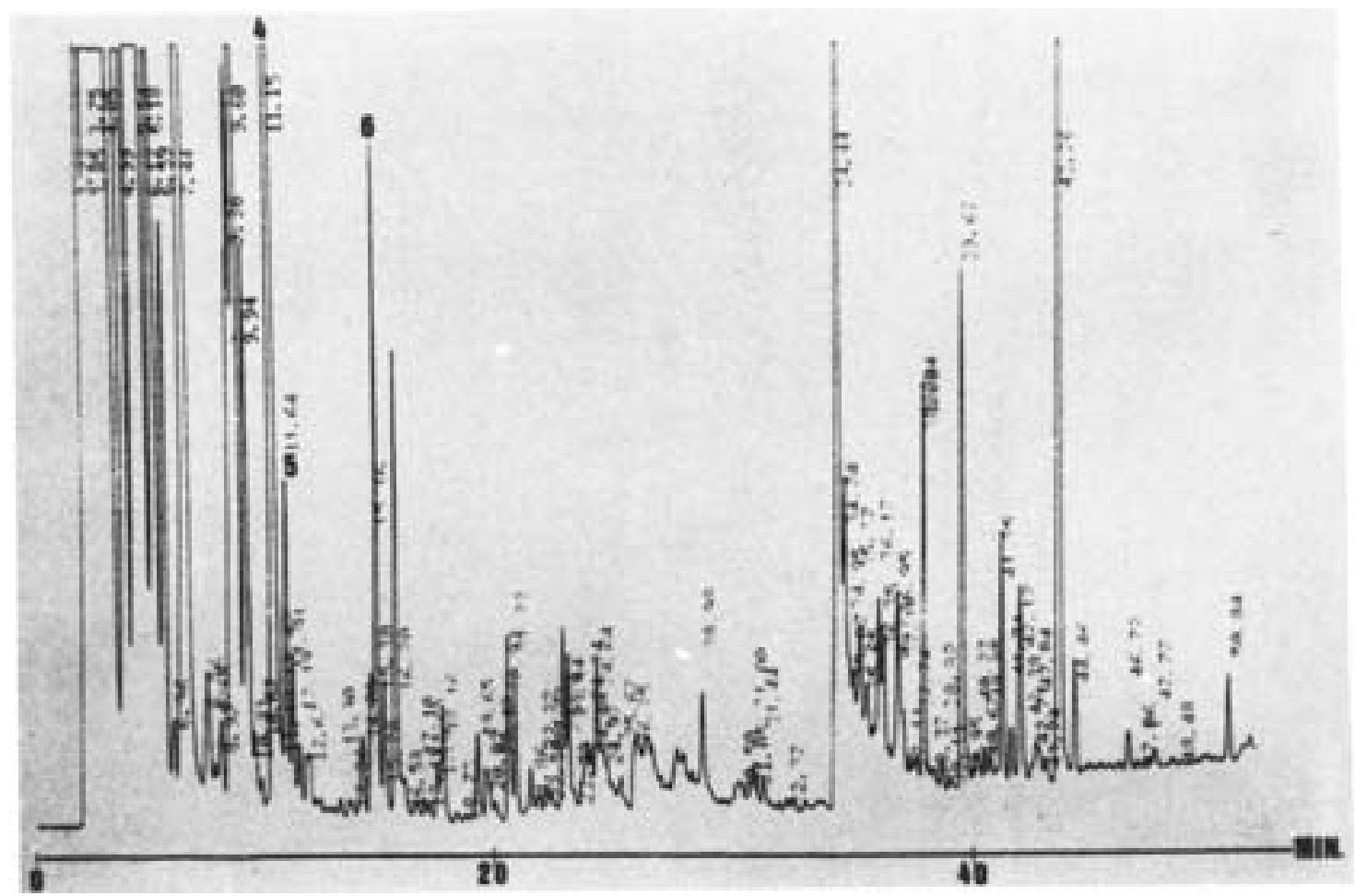

Figura 2. Perfil cromatogáfico de um café torrado de qualidade inferior (amostra D) obtido por "Headspace" - CGAR. Coluna Supelcowax 10, detector por ionização em chama, temperatura da coluna programada de $40^{\circ} \mathrm{C}$ a $190^{\circ} \mathrm{C}$ com taxa de $3^{\circ} \mathrm{C} / \mathrm{min}$., "split" de 1:20. Os picos 4 , 5 e 6 também sofreram tratamento estatístico através do "PCA".

fazer uma triagem dos picos relacionados à técnica de "Headspace-CGAR", devido a complexibilidade de seus cromatogramas. Utilizou-se como critério para a seleção desses picos o teste de reprodutibilidade. Este teste foi realizado através do cálculo dos coeficientes de variação das áreas desses picos para 10 replicatas de uma única amostra. Para a análise estatística só foram aproveitados os picos que apresentavam coeficiente de variação máximo de $15 \%^{3}$. Em seguida, tratou-se separadamente o conjunto das áreas dos picos dos cromatogramas, obtidos por cada uma das técnicas cromatográficas, com o "PCA". Dessa forma, obtivemos os picos mais representativos da variabilidade das amostras para cada um desses métodos cromatográficos. No caso da CEAE-UV, estes picos foram designados como picos 1 e 2, enquanto na CEAEIR como pico 3. Já para o "Headspace" - CGAR foram os picos 4,5 e 6 . As áreas destes seis picos selecionados (de cada uma das seis amostras) foram introduzidas simultaneamente no "PCA". A tabela 1 fornece o percentual de variância obtido pelo "PCA"para cada área. Pela análise desta tabela, podemos verificar que as três primeiras áreas contribuem com mais de $90 \%$ da variabilidade entre as seis amostras estudadas. Dessa forma, fica evidente que os dados obtidos pelas duas técnicas de CEAE são os principais parâmetros representativos da variabilidade do sistema.

Para evidenciar a dispersão das amostras, foi feito um gráfico de dispersão ("scatterplot") com os valores das duas principais áreas representativas dessa variabilidade (figura 3), visando diferenciar melhor as amostras em termos de qualidade. Pela análise do "scatterplot", pode-se verificar que a dispersão das amostras de café estudadas foi alta. Esta alta dispersão já havia sido detectada de modo mais acentuado, quando os dados fornecidos por cada uma das técnicas cromatográficas foram analisados isoladamente pelo "PCA". Entretanto, a tendência de agrupamento, de acordo com as características das amostras, deve se acentuar a medida que se aumente o tamanho da amostragem. Os resultados obtidos evidenciaram a influência da técnica de CEAE, particularmente da CEAE-UV,
Tabela 1. Percentual de variância para cada classe de área introduzida no "PCA".

\section{Principal Components Analysis}

$\begin{array}{ccc}\begin{array}{c}\text { Component } \\ \text { Number }\end{array} & \begin{array}{c}\text { Percent of } \\ \text { Variance }\end{array} & \begin{array}{r}\text { Cumulative } \\ \text { Percentage }\end{array} \\ 1 & 53.47950 & 53.47950 \\ 2 & 22.18557 & 75.66507 \\ 3 & 15.84256 & 91.50764 \\ 4 & 5.72509 & 97.23272 \\ 5 & 2.76728 & 100.00000 \\ 6 & .00000 & 100.00000\end{array}$

na variabilidade dos sistemas analisados. Além disso, essa técnica apresenta vantagens em relação a outras técnicas de cromatografia líquida de alta eficiência, como por exemplo, em relação a fase reversa. Segundo De Maria et $a l^{6}$, o método de CEAE é adequado para aplicação em análises de rotina, porque analisa simultaneamente o ácido clorogênico total, a trigonelina e a cafeína. Já a cromatografia em fase reversa analisa esses três componentes separadamente, porque as condições cromatográficas necessárias são diferentes. Além disso, no método de CEAE, usa-se somente água como fase móvel, o que é atrativo em termos de custo. Os perfis cromatográficos obtidos por cromatografia gasosa nas amostras de "headspace", mostram o potencial da mesma para o estudo, através do acoplamento da espectrometria de massas, de frações relevantes para o aroma. A identificação de compostos majoritários do café verde através de cromatografia líquida como descrita no presente trabalho mostra a importância dessa técnica para análise simultânea de compostos como sacarose, ácidos clorogêni$\cos$, trigonelina e cafeína, todos relacionados à qualidade final 


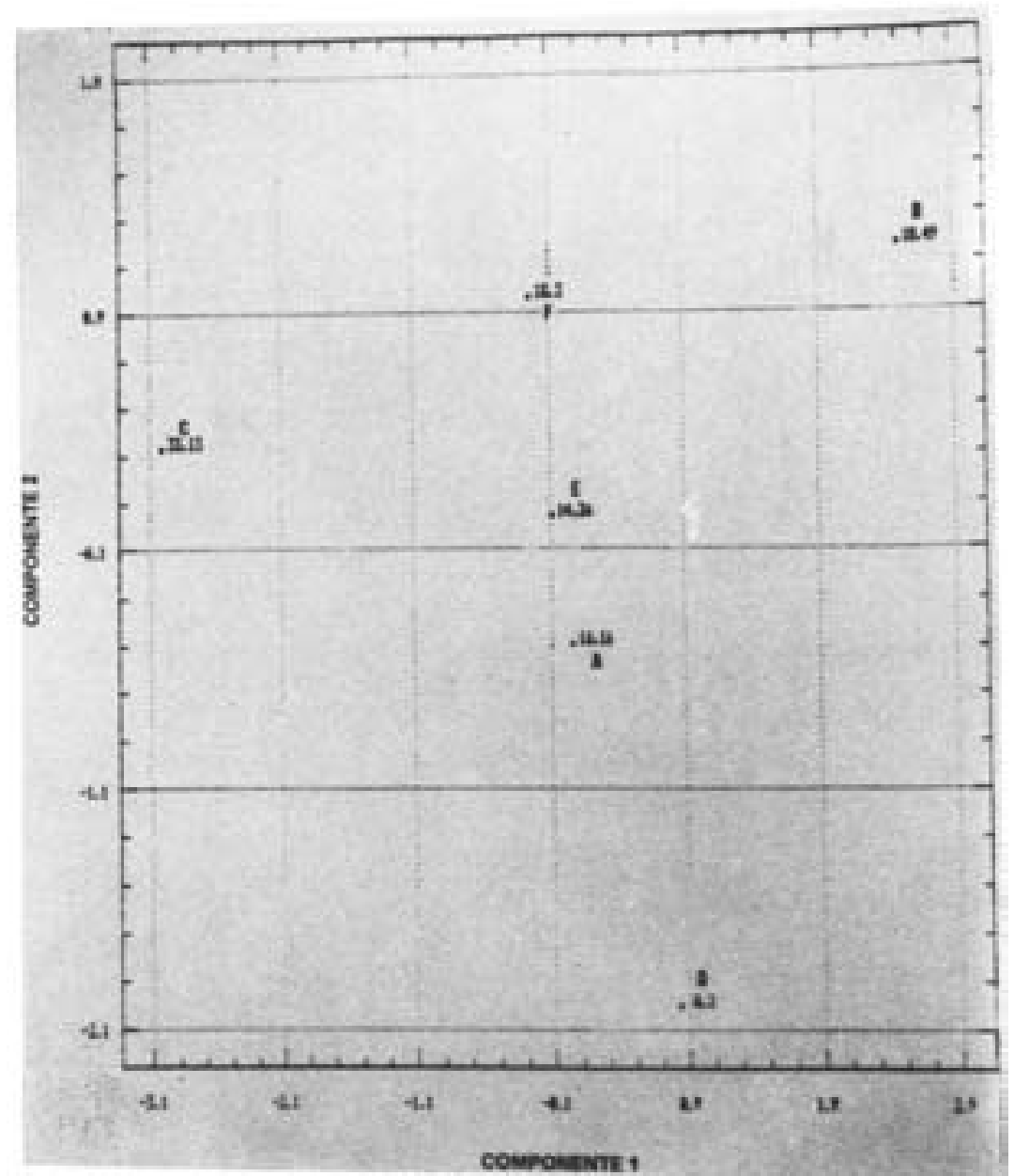

Figura 3. "Scatterplot" dos dois componentes principais. A, B, C - Cafés de boa qualidade. $\boldsymbol{D}, \boldsymbol{E}, \boldsymbol{F}-$ Cafés de péssima qualidade.

da bebida do café, quer seja pelos seus atributos organolépticos naturais, quer seja pela formação de produtos de degradação ou polimerização originando pigmentos e componentes voláteis da fração de aroma, após a torrefação. Os resultados obtidos no presente trabalho indicam a necessidade de estudos subsequentes, visando, por exemplo, a discriminação mais detalhada de áreas específicas na cromatografia de exclusão, bem como estudos mais detalhados de caracterização de compostos, através de técnicas complementares como a espectrometria de massa acoplada à cromatografia gasosa. Acreditamos que o avanço na utilização das técnicas básicas descritas aliado a utilização de uma amostragem mais significativa e complementadas com a análise por PCA poderão fornecer informações relevantes, que correlacionadas à análise sensorial, poderão ser instrumentos poderosos na caracterização da qualidade de amostras de café.

\section{AGRADECIMENTOS}

Agradecemos ao CNPq e a CAPES (Brasil) pelo suporte financeiro e à empressa ILLYCAFFÈ (Itália) pela doação das amostras e da coluna de CEAE utilizadas neste estudo.

\section{REFERÊNCIAS}

1. Bicchi, C. P.; Binello, A. E.; Legovich, M. M.; Pellegrino, G. M. and Vanni, A.; J. Agric. Food Chem. 1993, 41, 2324.

2. Dart, S. K. and Nursten, H. E.; Volatile components; In: Coffee Chemistry; Clarke and Macrae Eds.; London, 1985, vol. I, p. 223 - 265

3. Shimoda, M. and Shibamoto, T.; J. Agric. Food Chem. 1990, 38, 802 .

4. De Maria, C. A. B.; Trugo, L. C.; Moreira, R. F. A. and Werneck, C. C.; Food Chem. 1994, 49, 141.

5. STSC (Statistical Graphics System by Statistical Graphics Corporation); Statgraphics; Hewlett - Packard Corporation, 1986.

6. De Maria, C. A. B., Trugo, L. C. and Moreira, R. F. A.; Food Chem. 1995, 52, 447. 